\title{
Clusteroluminescence in Maleimide: from Well-Known Phenomenon to Unknown Mechanism
}

Yuan Xie, ${ }^{1,4,5}$ Dan Liu, ${ }^{1}$ Haoke Zhang, ${ }^{2,3 *}$ Dong Wang, ${ }^{4,5 *}$ Zheng Zhao, ${ }^{1 *}$ Ben Zhong Tang ${ }^{1,2,4 *}$ 1. Shenzhen Institute of Aggregate Science and TechnologySchool of Science and EngineeringThe Chinese University of Hong KongShenzhen 518172, China

2. MOE Key Laboratory of Macromolecular Synthesis and Functionalization, Department of Polymer Science and Engineering, Zhejiang University, Hangzhou 310027, China

3. ZJU-Hangzhou Global Scientific and Technological Innovation Center, Hangzhou 311215, China

4. Center for AIE Research, College of Materials Science and Engineering, Shenzhen University, Shenzhen 518060, China

5. College of Physics and Optoelectronic Engineering, Shenzhen University, Shenzhen 518060, China Email:zhanghaoke@zju.edu.cn,wangd@szu.edu.cn,zhaozheng@cuhk.edu.cn,tangbenz@cuhk.edu.cn

\begin{abstract}
:
Maleimide compounds have a long history related to colour reactions. It has been reported that maleimide yields bright colour when it reacts with basic reagents. Inspired by its colour formation, we investigated the maleimide polymer produced by an organic base 1,8diazabicyclo[5.4.0]undec-7-ene (DBU). The resultant polyimide contains exceptional fluorescence, which is virtually non-emissive in dilute solutions but exhibits strong pink emission $(430 \mathrm{~nm}$ and $580 \mathrm{~nm})$ in the concentrated solution and solid state. The intense colour comes from such a simple chemical structure, the unconventional fluorophore can be undoubtedly categorized into clusteroluminogens. By controlling the organic base amount, the intensity ratio of purple and orange-red emission can be controlled. The interaction between maleimide and base is rapid, and it can be achieved in the solid-solid interface or solid-gas interface. The aim herein is to highlight the recent observation and hold strong implications for further potential applications in anti-counterfeiting, gas sensors.
\end{abstract}




\section{Introduction:}

The development of organic fluorophores has revolutionized in various fields such as electronics, ${ }^{1-3}$ energy, ${ }^{4}$ and medicine. ${ }^{5-7}$ Organic fluorophores have better structure-property tunability, flexibility, and biocompatibility compared with inorganic light-emitting materials. To achieve efficient fluorescent emission performance, rational design of traditional organic fluorophores is often associated with large $\pi$ conjugation (e.g., double bond, triple bond, or aromatic ring), strong donor and acceptor functional groups ${ }^{8,9}$ However, fluorophores with planar molecular structures are suffered severely from the aggregation-caused quenching (ACQ) effect. Those molecules are highly emissive in solutions but become non-emissive in the aggregate state, which significantly impedes the development of fluorophores in practical applications. In 2001, Tang's group coined the concept of aggregation-induced emission (AIE) ${ }^{10}$ Luminescence materials with the AIE character (AIEgens) exhibit opposite features to the ACQ effect, that is, strong emission from the aggregated state, but no emission or weak emission in the solution state. AIEgens generally possess non-coplanar and rotational groups, preventing them from $\pi$ - $\pi$ stacking and limiting intramolecular rotation and vibration in the aggregate state. ${ }^{11,12}$

On the other hand, traditional AIE or ACQ luminogens containing large $\pi$-conjugated aromatic systems have been extensively explored, in which their luminescence generally resulted from the most favourable $\pi-\pi^{*}$ transition. ${ }^{13}$ Recent studies have demonstrated an uncommon phenomenon that some compounds devoid of a conjugated aromatic system can still emit light. ${ }^{14}$ Unlike aromatic ACQ or AIE luminogen, nonconventional luminophores comprise electron-rich heteroatoms with lone pair electron or isolated unsaturated bonds (e.g, $\mathrm{C}=\mathrm{C}, \mathrm{C}=\mathrm{O}$, $\mathrm{C}=\mathrm{N}, \mathrm{C} \equiv \mathrm{N}) .{ }^{15}$ These chromophores can form into a cluster and the luminescence is originated from $\mathrm{n}-\pi^{*}$ transition. The above-mentioned luminophores reveal similar AIE characteristics which show intrinsic emission in the concentrated or solid state. This emission mechanism is rationalized by the clusterization-triggered emission (CTE) mechanism. ${ }^{16}$ Clusteroluminogen is used to describe these special luminophores (CLgens). CLgens have gradually drawn many researchers' attention as they are not considered to be typical luminescent materials. ${ }^{17}$

Recent studies have revealed that many natural biomolecules such as bovine serum albumin (BSA), ${ }^{18}$ polypeptide,${ }^{19}$ non-aromatic amino acids displayed intrinsic luminescence, ${ }^{20,21}$ which are categorized into CLgens. Inspired by these natural biopolymers, many synthetic polymeric CLgens including poly(maleic anhydride-alt-vinyl acetate), ${ }^{22}$ poly(taconic anhydride-co-vinyl 
caprolactam) ${ }^{23}$ and polyester ${ }^{24}$ are reported in these few years. ${ }^{25}$ Nevertheless, the majority of CLgens are reported at the relatively short excitation wavelength $\left(\lambda_{\mathrm{ex}}<300 \mathrm{~nm}\right)$ with low emission efficiency $(\Phi) .{ }^{26,27}$ By converting small molecules to polymers, longer emission wavelength $\left(\lambda_{\mathrm{em}}\right)$ and higher emission efficiency $(\Phi)$ can be realized. ${ }^{23,28}$ Maleimide is a perfect candidate to conduct clusteroluminescence (CL) investigation as it is a simple, cheap heteroatom molecule with an aromatic free system and unsaturated bond, $\mathrm{C}=\mathrm{C}, \mathrm{C}=\mathrm{O}$ ). Even though maleimide moiety was regarded as a fluorescent quencher for many years, it was used to design for biological fluorescent probes through Michael addition. ${ }^{29}$ Our group recently reported that maleimide and succinimide derivatives can form a heterocyclic framework and emit light in the solid state, showing a strategy to facilitate the emission by increasing the possibility of the non-radiative $n-\pi^{*}$ transition and stabilizing the excitons in the excited state. ${ }^{27}$ Although the maximum emission wavelength was around 500nm, the remarkable emissive feature of maleimide small molecules inspires us to explore the emissive characteristic of their polymeric counterparts. A few previous works have revealed maleic anhydride-related copolymers with emissions ranging from blue to red. ${ }^{22,23,30,31}$ However, the inherent mechanism is still inexplicable, and copolymer was not the perfect paradigm to explore the fluorescence characteristics of that moiety.

Herein, inspired by a side reaction induced from thiol-maleimide Michael addition, ${ }^{32}$ we presented our recent endeavour to achieve multiple fluorescent emission of maleimide derivatives by introducing nucleophilic catalyst 1,8-diazabicyclo [5.4.0] undec-7-ene (DBU) to the maleimide monomer solution. The organic base acts as both a polymerization initiator and an external factor to induce fluorescence. The resultant polymer displayed pink emission with the combination of purple $\left(\lambda_{\mathrm{em}}=430 \mathrm{~nm}\right)$ and orange-red $\left(\lambda_{\mathrm{em}}=580 \mathrm{~nm}\right)$ light. By controlling the concentration of DBU, the ratio of blue and orange-red emission intensity can be modified to achieve white colour emission. More importantly, for the first time, the emission resulting from the maleimide and organic base can be even achieved in solid-solid or solid-gas interaction.

\section{Results and discussion:}

The initial search was inspired by thiol-maleimide end-group functionalization of reversible addition-fragmentation chain transfer (RAFT) polymer in the presence of nucleophile catalyst and polar solvents such as dimethyl sulfoxide (DMSO). Previous studies have shown that instead of functionalizing the maleimide end group in the polymer, the maleimide derivatives 
started to self-polymerize themselves through anionic polymerization in the presence of a nucleophile catalyst (such as amine). ${ }^{32,33}$ The solution instantaneously became dark red with an increased viscosity after 12 hours, which inspired us to investigate the colour origin and emission features. We selected three maleimide derivatives monomers including maleimide (MI), (n-ethyl maleimide(eMI)), n-phenyl maleimide(pMI) and DBU as a nucleophilic catalyst to study the effect of polymerization time, functional groups on the emission characteristics. The solution-state absorption and PL spectra were measured to compare the monomer and polymer state of three MI derivatives (Figure 1). Three MI derivatives display the same trend. Both absorption and emission spectra demonstrated that the MI polymers displayed significantly stronger UV absorption and fluorescence emission than that of their monomeric counterparts, but the intensity varies between the three MI polymeric derivatives. All the MI polymers display excitation-dependent emission peaks at $430 \mathrm{~nm}\left(\lambda_{\mathrm{ex}}=400 \mathrm{~nm}\right)$ and $580 \mathrm{~nm}$ $\left(\lambda_{\mathrm{ex}}=490 \mathrm{~nm}\right)$ without large bathochromic or hypsochromic shifted, suggesting the emitter center could be the same one. However, the emission of eMIP is much weaker than others (Figure 1E), this could be associated with the flexible ethyl group in the eMIP structure, which typically relaxes via a nonradiative decay pathway upon excitation, leading to weaker fluorescence emission. The nearly invisible photo of eMIP powder in figure 1E under UV irradiation $\left(\lambda_{\mathrm{ex}}=400 \mathrm{~nm}\right.$ ) further confirms this.

Alternatively, MIP shows stronger emission peaks at 430nm than pMIP in the solution, whereas pMIP displays enhanced emission peaks at $580 \mathrm{~nm}$ (Figure 1B, C, H, I). The pMIP solid state also exhibits much redder form than MIP. This could be due to the phenyl substituted group in the pMIP structure. All the phenyl groups in the polymer chain are spatially close to each other, which increases the population of through-space electron delocalization, rendering a much redder emission feature. Additionally, even though recent reports have shown that MI monomer displayed fluorescence emission around 456nm in the concentrated or solid state, the fluorescence of the monomer solution under this condition is still much weaker compared to the polymeric one. 

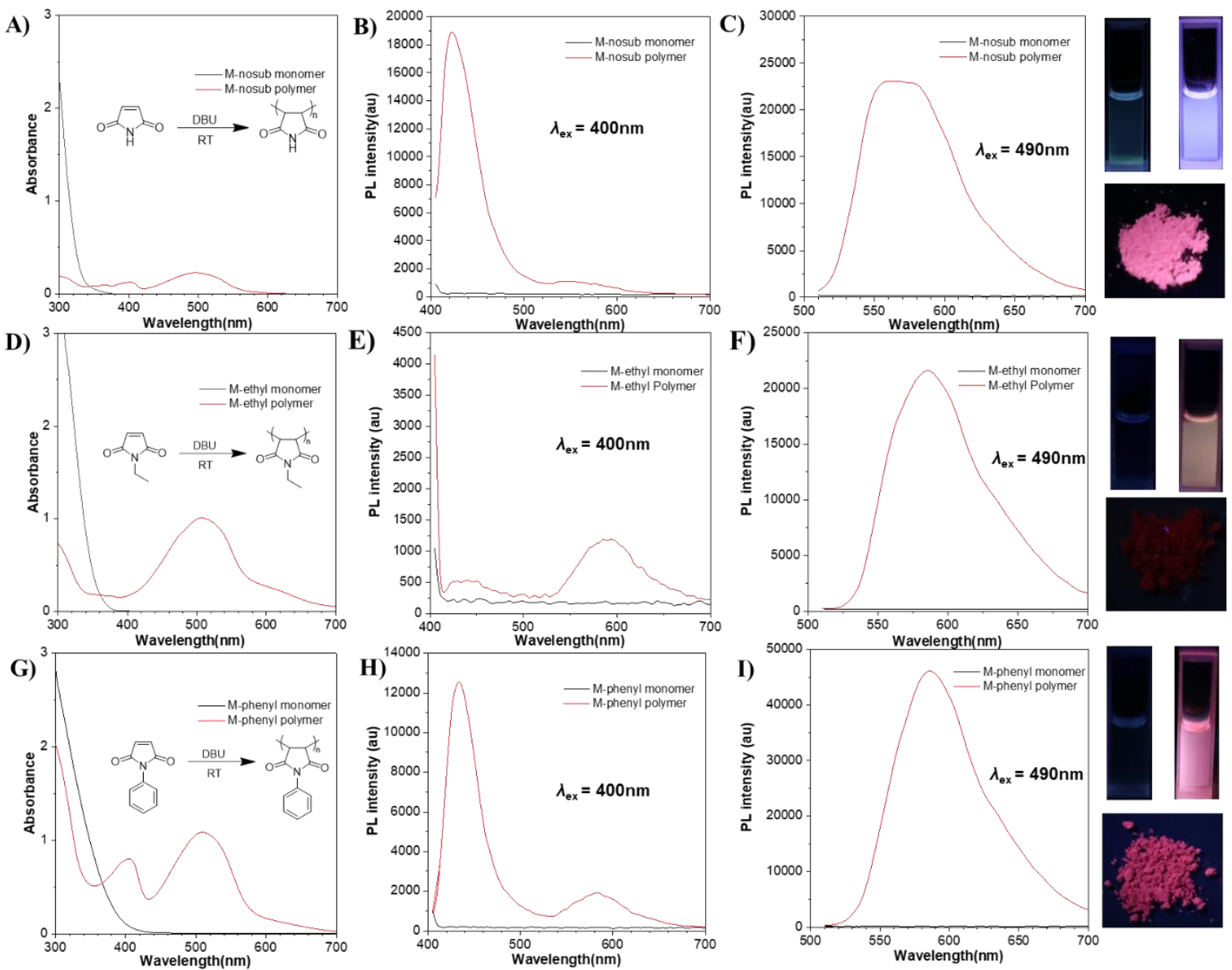

Figure 1. A) UV-vis absorption of $5 \mathrm{mM}$ maleimide monomer(MIM) and maleimide polymer(MIP) solution in dimethyl sulfoxide (DMSO); B) Photoluminescence (PL) spectra of MIM and MIP solution in DMSO ( $c=5 \mathrm{mM}$, $\left.\lambda_{e x}=400 \mathrm{~nm}\right)$; C) PL spectra of maleimide monomer and maleimide polymer solution in DMSO $\left(c=5 \mathrm{mM}, \lambda_{e x}=\right.$ $490 \mathrm{~nm}$ ), photo taken under $365 \mathrm{~nm}$ UV irradiation, MIM solution (top left), MIP solution(top right), MIP powder(bottom); D) UV-vis absorption $(c=5 \mathrm{mM})$ of n-ethyl maleimide monomer(eMIM) and n-ethyl maleimide polymer(eMIP) solution in DMSO; E) PL spectra of eMIM and eMIP solution in DMSO ( $\left.c=5 \mathrm{mM}, \lambda_{e x}=400 \mathrm{~nm}\right)$; F) PL spectra of eMIM and eMIP solution in DMSO $\left(c=5 \mathrm{mM}, \lambda_{e x}=490 \mathrm{~nm}\right)$, photo taken under $365 \mathrm{~nm}$ UV irradiation, eMIM solution (top left), eMIP solution(top right), eMIP powder(bottom); G) UV-vis absorption ( $c$ $=5 \mathrm{mM})$ of $\mathrm{n}$-phenyl maleimide monomer(pMIM) and n-phenyl maleimide polymer(pMIP) solution in DMSO; H) PL spectra of pMIM and pMIP solution in DMSO $\left(c=5 \mathrm{mM}, \lambda_{e x}=400 \mathrm{~nm}\right)$; I) PL spectra of pMIM and pMIP solution in DMSO ( $c=5 \mathrm{mM}, \lambda_{e x}=490 \mathrm{~nm}$ ), photo taken under $365 \mathrm{~nm}$ UV irradiation, pMIM solution (top left), pMIP solution(top right), pMIP powder(bottom);

In order to investigate the effect of polymerization time on the MIP absorption and emission, we conducted time-dependent absorption and PL emission monitoring through MI polymerization at different time intervals. Before adding DBU into the monomer solution, MI monomer does not display any absorption before 400nm, and PL shows a negligible emission range between 400 and $700 \mathrm{~nm}$ (Figure $2 \mathrm{~A}, \mathrm{C}, \mathrm{D}$ ). After adding the DBU, the absorption and 
PL of the solution started to increase gradually over time. The absorption spectra presented a gradual increase from 0 mins to 60 mins and were maintained at the level after 60 mins (Figure 2A). The pattern of gradually increasing was also observed in the PL spectra at the short excitation wavelength $\left(\lambda_{\mathrm{ex}}=400 \mathrm{~nm}\right.$ ) (Figure $\left.2 \mathrm{~B}\right)$. it is interesting to note that both emission peaks $(430 \mathrm{~nm}$ and $580 \mathrm{~nm}$ ) appeared progressively under the condition (50mM of MI monomer) (Figure 2C), which is not the same case in Figure 1B, where the emission peak at $420 \mathrm{~nm}$ is much stronger than peaks at $580 \mathrm{~nm}$ at the short excitation wavelength. The difference is that the solution is prepared from $0.5 \mathrm{M}$ of $\mathrm{MI}$ monomer in the former case with the same amount of DBU initiator and diluted into 50mM. The time-dependent measurement of $0.5 \mathrm{M}$ MI solution was also collected under the same condition (Figure S1B), which matched the results in figure 1B. The underlying mechanism is still under investigation. With the condition of 50mM MI solution, the emission peaks at 430nm and 580nm merged after 1 day and become white colour emission, evidenced by the photo (Figure 2C, inset). However, the emission peak at $580 \mathrm{~nm}$ achieved its maximum at 15mins and showed a slight decline after that (Figure 2D). This is because the solution showed a broad absorption peak from almost 600 to $400 \mathrm{~nm}$ and increased over time, giving rise to a significant overlap between absorption and emission spectra. As a result, the light released from MIP is expected to be reabsorbed by itself, attenuating its intrinsic fluorescence intensity.

On the other hand, the emission exhibits excitation and solvent-dependent wavelength. By varying the excitation wavelength from 400 to $450 \mathrm{~nm}$, the emission covers the visible light spectrum from 400 to $650 \mathrm{~nm}$. The PL emission started to narrow down to yellow and orangered at excitation wavelengths from 460 to 510nm (Figure 2B), indicating its CL feature. Both MIP and pMIP polymer were used to conduct its solvent-dependent measurement. Under different solvents systems, it is worth noting that the fluorescence emission did not display any red or blue-shifted as conventional AIEgens do, but differed in its intensity, which could be due to its solubility in the corresponding solvents. both MIP and pMIP can only be dissolved in the polar aprotic solvent such as DMF, DMSO, and MeCN (Figure 3A), but pMIP has better solubility than MIP and it does not completely participate in most common organic solvents because of the phenyl substitute. Due to the difference in solubility, the PL emission behaves differently in the solvents. It can be concluded that protic solvents such as water and methanol have a detrimental effect on fluorescence emission of both MIP and pMIP with the lowest PL intensity. Water and methanol as a proton source can disturb the cluster complexes from the polymer and DBU. On the other hand, both polarity and solubility together contribute 
significantly to the PL emission of the polymers. The highest PL emission can be achieved in non-polar solvents in 1,4 dioxane, THF, chloroform and DCM. However, MIP basically participated in DCM and chloroform, which can be visually seen in figure 4A. Although MIP is also not dissolved in 1,4 dioxane, THF, it forms a cluster, enhancing its luminescence, further suggesting its CTE features. DMSO and DMF can dissolve polymers with relatively high PL intensity as DMSO and DMF contain heteroatoms, which can stabilize the excited state.

A)
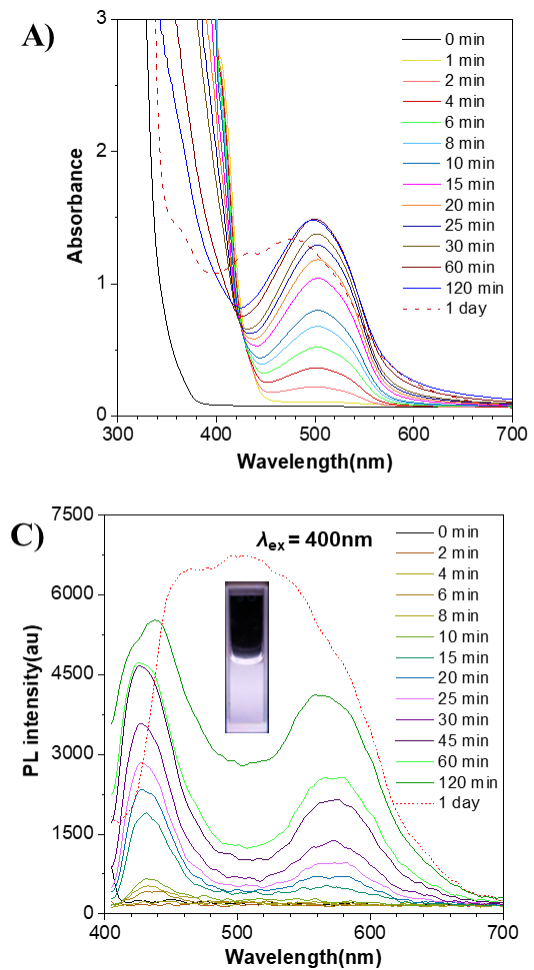

B)

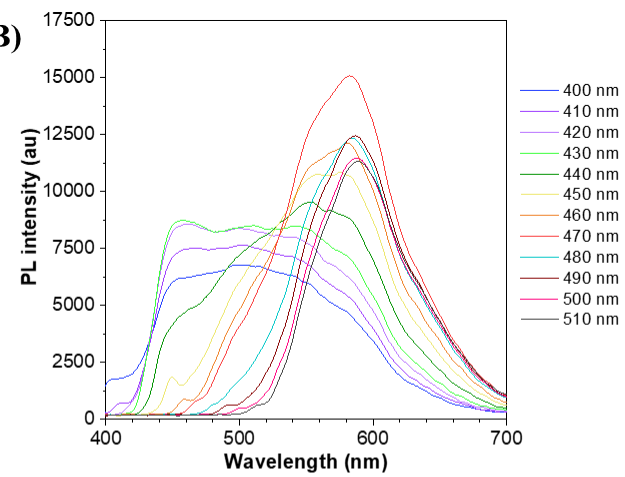

D)



Figure 2. A) UV-vis absorption of 50mM maleimide monomer (MIM)+10ul DBU in dimethyl sulfoxide (DMSO) at the different time interval; B) Photoluminescence (PL) spectra of 50mM MIP solution in DMSO at various excitation wavelength; C) PL spectra of 50mM maleimide monomer (MIM)+10ul DBU in DMSO at the different time interval $\left(\lambda_{\mathrm{ex}}=400 \mathrm{~nm}\right)$, inset photo was taken under $365 \mathrm{~nm}$ UV irradiation; D) PL spectra of 50mM maleimide monomer (MIM)+10ul DBU in DMSO at the different time interval $\left(\lambda_{\mathrm{ex}}=490 \mathrm{~nm}\right)$. 

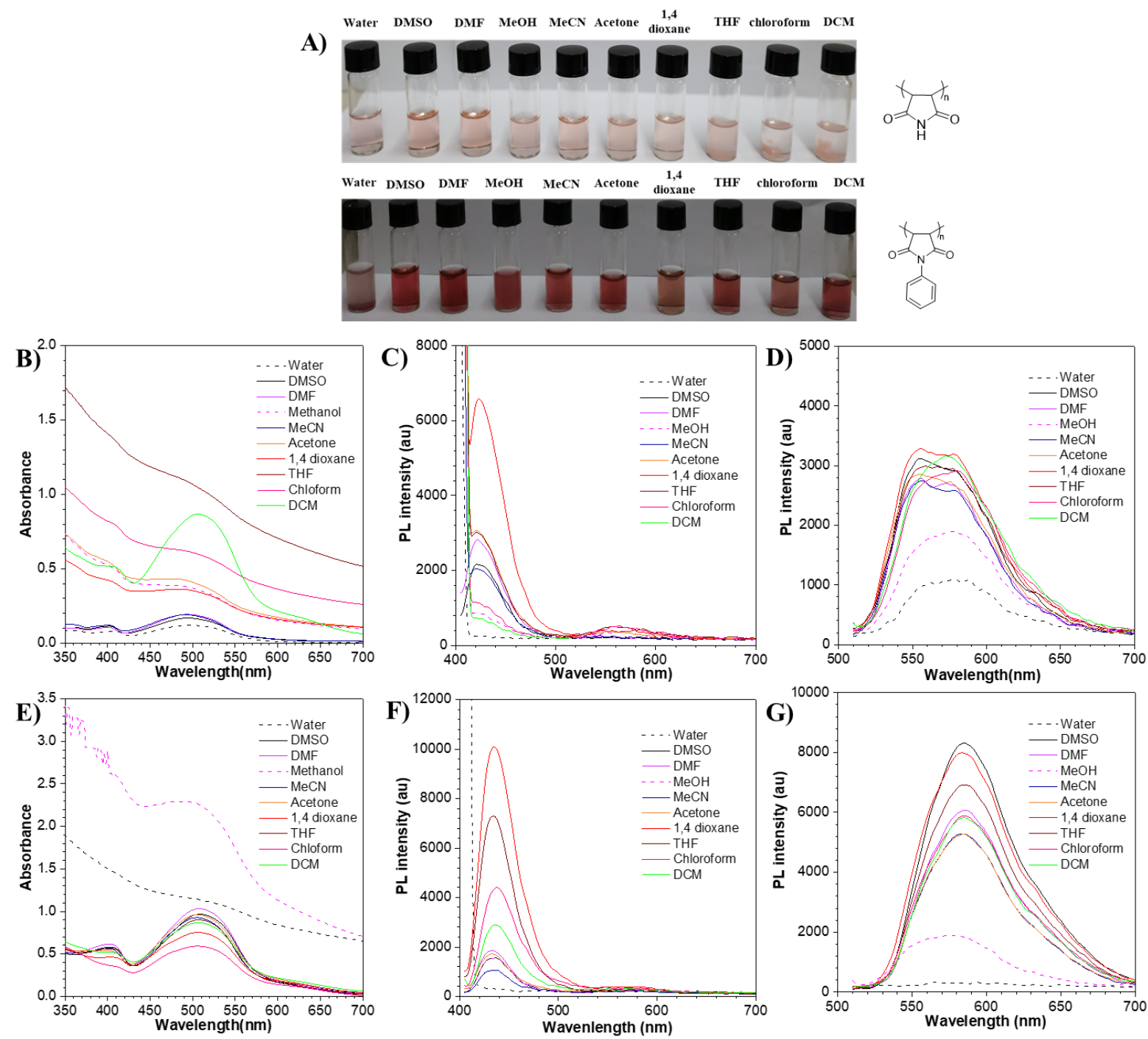

Figure 4. A) photo of maleimide (MIP) (top) and n-phenyl maleimide(pMIP) (bottom) polymers dissolved in various solvents B) UV-vis absorption of $5 \mathrm{mM}$ MIP solution in the different solvents systems. C) photoluminescence (PL) spectra of 5mM MIP polymer solution in the different solvent systems $\left(\lambda_{e x}=400 \mathrm{~nm}\right)$. D) PL spectra of 5mM MIP polymer solution in the different solvent systems ( $\left.\lambda_{\mathrm{ex}}=490 \mathrm{~nm}\right)$. E) UV spectra of $5 \mathrm{mM}$ pMIP solution in the different solvent systems. E) PL spectra of $5 \mathrm{mM}$ pMIP polymer solution in the different solvent systems $\left(\lambda_{e x}=400 \mathrm{~nm}\right)$. F) E) PL spectra of $5 \mathrm{mM}$ pMIP polymer solution in the different solvent systems $\left(\lambda_{e x}=490 \mathrm{~nm}\right)$.

To further explore the fluorescence origin induced from poly (maleimide) and DBU, we eliminate the external factor by selecting poly (n-phenyl maleimide) (PPM) prepared from free radical polymerization and n-phenyl maleimide monomer(pMIM). The PPM prepared from free radical polymerization was to eliminate the DBU. Additionally, we chose to mix PPM powder or pMIM with solid organic base 4- dimethylaminopyridine(DMAP) in the absence of solvent. PPM powder prepared from free radical polymerization was pale white powder without strong red emission under UV lamp, whereas the pMIP powder prepared from DBU 
induced polymerization was red with the same precipitation treatment, suggesting DBU not only act as a polymerization initiator, but some other interaction with the polymer to initiate fluorescence. The pink colour immediately appeared when PPM is mixed with DMAP (molar ratio 1:1) in a mortar and pestle. The pink colour started to become brighter and covered the whole mixture after $5 \mathrm{mins}$. After $10 \mathrm{mins}$, there was no colour change in the mixture, indicating the interaction between PPM and DMAP is rapid even in the solid-solid interface. Then, the MI monomer was mixed with DMAP in the same molar ratio to explore whether the interaction is only restricted with maleimide polymer. Using the same mixing method, the fluorescent colour was orange and the time to appear the colour was 1 day, which was much longer than that of that polymer counterpart. This may be due to the C-C double bond in the MI. However, the underlying mechanism is still investigating.

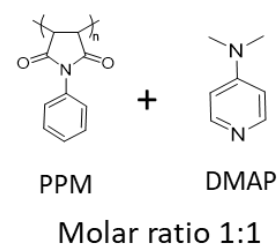

A)

Initial

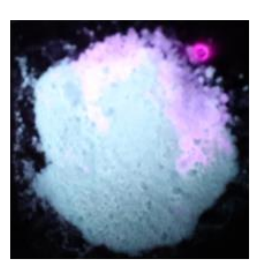

B)

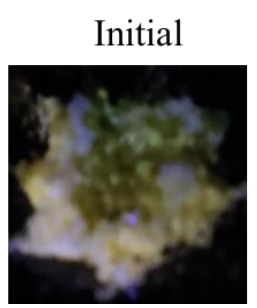

$5 \min$


$5 \min$

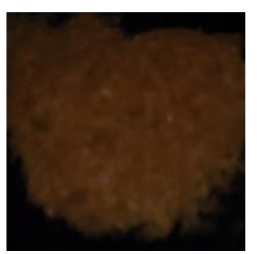

1hour

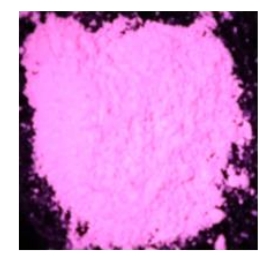

1hour



1 day

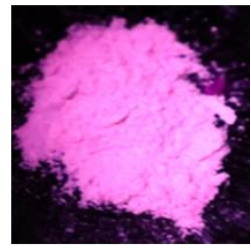

Molar ratio $1: 1$ $10 \mathrm{~min}$
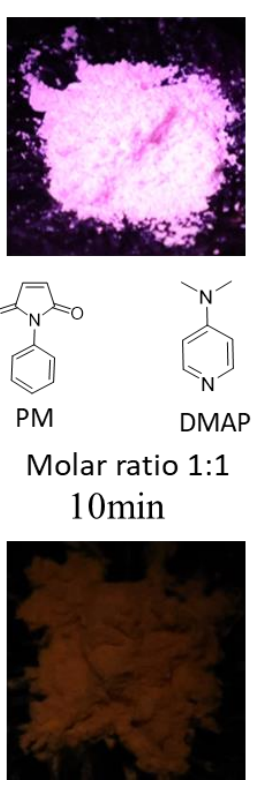

MAP

Figure 4. A) photo was taken under $365 \mathrm{~nm}$ UV irradiation. Poly (n-phenyl maleimide) (PPM) (prepared from free radical polymerization) mixed with dimethylaminopyridine (DMAP) in a mortar and pestle at the different time intervals (molar ratio 1:1); B) photo was taken under $365 \mathrm{~nm}$ UV irradiation. n-phenyl maleimide monomer mixed with dimethylaminopyridine (DMAP) in a mortar and pestle at the different time intervals (molar ratio 1:1).

The interaction between PPM and DMAP was rapid in the solid-solid interface. We then try to explore if the interaction can be achieved in the solid-gas interface. The PPM powder was 
exposed to triethylamine (TEA) in a container and leave it for 30 mins. The PPM powder turned to red and showed the pink fluorescent colour in the solid state, which may suggest the interaction in the solid-solid interface is the same as that in the solid-gas interface. The PPM powder was then exposed to $\mathrm{HCl}$ gas, and the luminescence was reversed to its original form after being exposed to $\mathrm{HCl}$ gas. The powder gives rise to a luminescence on-off responsive cycle upon exposure to acid or base vapour. The cycle was continued to 3 cycles, indicating the interaction is highly reversible (Figure 5). The powder can be potentially fabricated into test strip or solid-state kit for potential sensor applications.

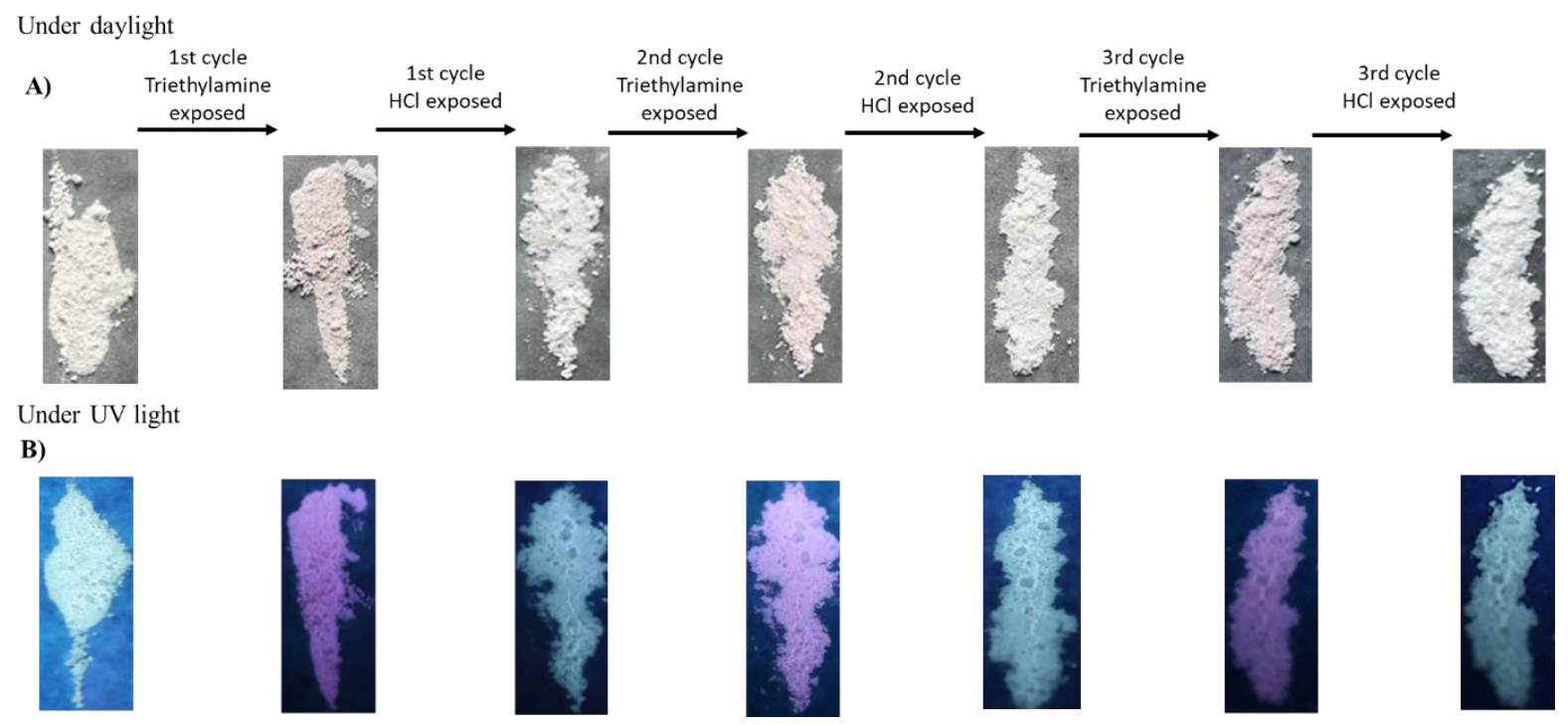

Figure 5. A) Photo was taken at daylight when poly (n-phenyl maleimide) prepared from free radical polymerization is under triethylamine (TEA) gas exposure then $\mathrm{HCl}$ gas exposure for 3 cycles at room temperature; B) Photo was taken under 365 UV irradiation when PPM is exposed with TEA gas, then $\mathrm{HCl}$ gas for 3 cycles at room temperature.

\section{Conclusion:}

New insights into maleimide as a luminescence emitter have been provided. DBU acts as both a polymerization initiator and an external factor to induce purple and red fluorescence emission at $430 \mathrm{~nm}$ and 580nm. By controlling the DBU amount, the ratio of purple light and orange-red can be controlled and even merged both $430 \mathrm{~nm}$ and $580 \mathrm{~nm}$ peaks together to achieve white light emission. Poly(maleimide) prepared from free radical polymerization does not exhibit the same emission as the polymer prepared from DBU induced anionic polymerization. However, it can interact rapidly with a solid organic base DMAP to emit solid state fluorescence in the absence of solvents. Additionally, the polymer can achieve multiple reversible fluorescence 
switches in the solid-gas interaction when exposed to TEA and $\mathrm{HCl}$ gas. This new phenomenon enables various potential applications and mechanism investigation in the future.

\section{Acknowledgment:}

The work was partially supported by the National Natural Science Foundation of China (52122317, 22175120, 52003228). The Science and Technology Plan of Shenzhen ( JCYJ20210324134613038) and Open Fund of Guangdong Provincial Key Laboratory of Luminescence from Molecular Aggregates (2019B030301003). H. Z. is thankful for the support from the Fundamental Research Funds for the Central Universities (2021QNA4032)

\section{References:}

1. Cai, Z. Y.; Wu, X.; Liu, H.; Guo, J. J.; Yang, D. Z.; Ma, D. G.; Zhao, Z. J.; Tang, B. Z., Realizing Record-High Electroluminescence Efficiency of $31.5 \%$ for Red Thermally Activated Delayed Fluorescence Molecules. Angew Chem Int Edit 2021, 60 (44), 23635-23640.

2. Li, Q.; Li, Z., Molecular Packing: Another Key Point for the Performance of Organic and Polymeric Optoelectronic Materials. Accounts of Chemical Research 2020, 53 (4), 962-973.

3. Fu, Y.; Liu, H.; Yang, D.; Ma, D.; Zhao, Z.; Tang, B. Z., Boosting external quantum efficiency to $38.6 \%$ of sky-blue delayed fluorescence molecules by optimizing horizontal dipole orientation. 2021, 7 (43), eabj2504.

4. Robertson, L. A.; Shkrob, I. A.; Agarwal, G.; Zhao, Y.; Yu, Z.; Assary, R. S.; Cheng, L.; Moore, J. S.; Zhang, L., Fluorescence-Enabled Self-Reporting for Redox Flow Batteries. ACS Energy Letters 2020, $5(9), 3062-3068$.

5. Zhu, S.; Tian, R.; Antaris, A. L.; Chen, X.; Dai, H., Near-Infrared-II Molecular Dyes for Cancer Imaging and Surgery. Advanced Materials 2019, 31 (24), 1900321.

6. Mei, J.; Leung, N. L. C.; Kwok, R. T. K.; Lam, J. W. Y.; Tang, B. Z., Aggregation-Induced Emission: Together We Shine, United We Soar! Chemical Reviews 2015, 115 (21), 11718-11940.

7. Carr, J. A.; Franke, D.; Caram, J. R.; Perkinson, C. F.; Saif, M.; Askoxylakis, V.; Datta, M.; Fukumura, D.; Jain, R. K.; Bawendi, M. G.; Bruns, O. T., Shortwave infrared fluorescence imaging with the clinically approved near-infrared dye indocyanine green. 2018, 115 (17), 4465-4470.

8. Huang, X.; Han, S.; Huang, W.; Liu, X., Enhancing solar cell efficiency: the search for luminescent materials as spectral converters. Chemical Society Reviews 2013, 42 (1), 173-201.

9. Zhou, J.; Liu, Q.; Feng, W.; Sun, Y.; Li, F., Upconversion Luminescent Materials: Advances and Applications. Chemical Reviews 2015, 115 (1), 395-465.

10. Luo, J.; Xie, Z.; Lam, J. W. Y.; Cheng, L.; Chen, H.; Qiu, C.; Kwok, H. S.; Zhan, X.; Liu, Y.; Zhu, D.; Tang, B. Z., Aggregation-induced emission of 1-methyl-1,2,3,4,5-pentaphenylsilole. Chemical Communications 2001, (18), 1740-1741.

11. Tu, Y. J.; Zhao, Z.; Lam, J. W. Y.; Tang, B. Z., Aggregate Science: Much to Explore in the Meso World. Matter-Us 2021, 4 (2), 338-349.

12. Zhao, Z.; Zhang, H. K.; Lam, J. W. Y.; Tang, B. Z., Aggregation-Induced Emission: New Vistas at the Aggregate Level. Angew Chem Int Edit 2020, 59 (25), 9888-9907.

13. Frath, D.; Massue, J.; Ulrich, G.; Ziessel, R., Luminescent Materials: Locking $\pi$-Conjugated and Heterocyclic Ligands with Boron(III). Angewandte Chemie International Edition 2014, 53 (9), 22902310.

14. Zhang, H. K.; Zhao, Z.; McGonigal, P. R.; Ye, R. Q.; Liu, S. J.; Lam, J. W. Y.; Kwok, R. T. K.; Yuan, W. Z.; Xie, J. P.; Rogach, A. L.; Tang, B. Z., Clusterization-triggered emission: Uncommon luminescence from common materials. Mater Today 2020, 32, 275-292. 
15. Tang, S.; Yang, T.; Zhao, Z.; Zhu, T.; Zhang, Q.; Hou, W.; Yuan, W. Z., Nonconventional luminophores: characteristics, advancements and perspectives. Chemical Society Reviews 2021, 50 (22), 12616-12655.

16. Zhang, H. K.; Tang, B., Through-Space Interactions in Clusteroluminescence. Jacs Au 2021, 1 (11), 1805-1814.

17. Liao, P.; Huang, J.; Yan, Y.; Tang, B. Z., Clusterization-triggered emission (CTE): one for all, all for one. Materials Chemistry Frontiers 2021, 5 (18), 6693-6717.

18. Wang, Q.; Dou, X. Y.; Chen, X. H.; Zhao, Z. H.; Wang, S.; Wang, Y. Z.; Sui, K. Y.; Tan, Y. Q.; Gong, Y. Y.; Zhang, Y. M.; Yuan, W. Z., Reevaluating Protein Photoluminescence: Remarkable Visible Luminescence upon Concentration and Insight into the Emission Mechanism. Angew Chem Int Edit 2019, 58 (36), 12667-12673.

19. Zhang, Z.; Yan, W.; Dang, D.; Zhang, H.; Sun, J. Z.; Tang, B. Z., The role of amide $(n, \pi *)$ transitions in polypeptide clusteroluminescence. Cell Reports Physical Science 2022, 3 (2), 100716.

20. Chen, X.; Luo, W.; Ma, H.; Peng, Q.; Yuan, W. Z.; Zhang, Y., Prevalent intrinsic emission from nonaromatic amino acids and poly(amino acids). Science China Chemistry 2018, 61 (3), 351-359.

21. Ravanfar, R.; Bayles, C. J.; Abbaspourrad, A., Structural Chemistry Enables Fluorescence of Amino Acids in the Crystalline Solid State. Crystal Growth \& Design 2020, 20 (3), 1673-1680.

22. Zhao, E.; Lam, J. W. Y.; Meng, L. M.; Hong, Y.; Deng, H. Q.; Bai, G. X.; Huang, X. H.; Hao, J. H.; Tang, B. Z., Poly[(maleic anhydride)-alt-(vinyl acetate)]: A Pure Oxygenic Nonconjugated Macromolecule with Strong Light Emission and Solvatochromic Effect. Macromolecules 2015, 48 (1), 64-71.

23. Shang, C.; Zhao, Y.; Long, J.; Ji, Y.; Wang, H., Orange-red and white-emitting nonconventional luminescent polymers containing cyclic acid anhydride and lactam groups. J Mater Chem C 2020, 8 (3), 1017-1024.

24. Chu, B.; Zhang, H.; Hu, L.; Liu, B.; Zhang, C.; Zhang, X.; Tang, B. Z., Altering Chain Flexibility of Aliphatic Polyesters for Yellow-Green Clusteroluminescence in $38 \%$ Quantum Yield. Angewandte Chemie International Edition 2022, 61 (6), e202114117.

25. Liu, L.; Zhang, H. K.; Liu, S. J.; Sun, J. Z.; Zhang, X. H.; Tang, B. Z., Polymerization-induced emission. Mater Horiz 2020, 7 (4), 987-998.

26. Zhou, Q.; Yang, T.; Zhong, Z.; Kausar, F.; Wang, Z.; Zhang, Y.; Yuan, W. Z., A clusteringtriggered emission strategy for tunable multicolor persistent phosphorescence. Chemical Science 2020, 11 (11), 2926-2933.

27. He, B. Z.; Zhang, J.; Zhang, J. Y.; Zhang, H. K.; Wu, X. Y.; Chen, X.; Kei, K. H. S.; Qin, A. J.; Sung, H. H. Y.; Lam, J. W. Y.; Tang, B. Z., Clusteroluminescence from Cluster Excitons in Small Heterocyclics Free of Aromatic Rings. Adv Sci 2021, 8 (7).

28. Zhou, X.; Luo, W.; Nie, H.; Xu, L.; Hu, R.; Zhao, Z.; Qin, A.; Tang, B. Z., Oligo(maleic anhydride)s: a platform for unveiling the mechanism of clusteroluminescence of non-aromatic polymers. J Mater Chem C 2017, 5 (19), 4775-4779.

29. Chen, M. Z.; Moily, N. S.; Bridgford, J. L.; Wood, R. J.; Radwan, M.; Smith, T. A.; Song, Z. G.; Tang, B. Z.; Tilley, L.; Xu, X. H.; Reid, G. E.; Pouladi, M. A.; Hong, Y. N.; Hatters, D. M., A thiol probe for measuring unfolded protein load and proteostasis in cells. Nat Commun 2017, 8.

30. Guo, Z. Y.; Ru, Y.; Song, W. B.; Liu, Z. J.; Zhang, X. H.; Qiao, J. L., Water-Soluble Polymers with Strong Photoluminescence through an Eco-Friendly and Low-Cost Route. Macromol Rapid Comm 2017, 38 (14).

31. Hu, C. X.; Ru, Y.; Guo, Z. Y.; Liu, Z. J.; Song, J. H.; Song, W. B.; Zhang, X. H.; Qiao, J. L., New multicolored AIE photoluminescent polymers prepared by controlling the $\mathrm{pH}$ value. J Mater Chem $\mathrm{C}$ 2019, 7 (2), 387-393.

32. Abel, B. A.; McCormick, C. L., "One-Pot" Aminolysis/Thiol-Maleimide End-Group Functionalization of RAFT Polymers: Identifying and Preventing Michael Addition Side Reactions. Macromolecules 2016, 49 (17), 6193-6202. 
33. Azechi, M.; Toyota, N.; Yamabuki, K.; Onimura, K.; Oishi, T., Anionic polymerization of Nsubstituted maleimide with achiral and chiral amines as an initiator. Polym Bull 2011, 67 (4), 631-640. 